\section{Phase II and Phase III attrition rates 2011-2012}

Knowledge of the rates and causes of drug candidate attrition by clinical development phase and by therapeutic area is valuable in assessing the impact of changes in development strategy and research area focus by the pharmaceutical industry. Two years ago, we published brief reports on failures in Phase III and submission from 2007 to 2010 (Nature Rev. Drug Discov. 10, 87; 2011) and failures in Phase II from 2008 to 2010 (Nature Rev. Drug Discov. 10, 328-329; 2011). Here, using data gathered from Drugs of Today, as in the previous analyses, we have conducted a similar analysis for 2011 and 2012.

During this period, there were a total of 148 failures between Phase II and submission (also including Phase I/II studies in patients and major new indications of already marketed drugs). Of these, 105 had reported reasons for failure. The majority were due to a lack of efficacy (56\%) or to safety issues (28\%); here, safety includes those failures that were due to an insufficient therapeutic index (FIG. 1a).

When looked at by phase, for the most recent year range, the proportion of failures due to lack of efficacy was higher in Phase II (59\%), but still disturbingly high in Phase III and beyond (52\%). The proportion of failures due to safety issues is higher in Phase III and beyond compared with Phase II - at $35 \%$ and $22 \%$, respectively - which may be due to safety issues that only become apparent in larger numbers of patients and/or longer trials.

A comparison of the Phase II attrition rates with those reported 2 years ago shows a decrease in strategic and commercial failures (from $29 \%$ down to $16 \%$ ) with a concomitant increase in efficacy and safety failures (FIG. 1 b). A comparison for Phase III and beyond reveals an increase in safety failures (35\% up from $21 \%$ ) with a concomitant decrease in efficacy failures (FIG. 1 b).

When the failure rates are broken down by therapeutic area, oncology and central nervous system (CNS) disorders account for $44 \%$ (30\% and $14 \%$, respectively) of all the 105 failures between Phase II and submission for which reasons have been reported (FIG. 1a). However, almost $50 \%$ of CNS and endocrinology (diabetes) failures ( 13 out of 29 , and 4 out of 8 , respectively) are excluded from these numbers because the reason for the failure has not been disclosed. Oncology and CNS are areas in which it can be difficult to establish clear efficacy signals in small or short-duration Phase II trials, which reinforces the need to design trials that can deliver data that are sufficient to support good decision-making, and to have suitably discriminatory proof-of-concept criteria agreed prospectively.

Trend analysis from the CMR International Global R\&D Performance Metrics Program of new development projects across a group of 16 companies (representing approximately $65 \%$ of global RED expenditure) reveals that the Phase II success rates for new development projects remained below $20 \%$ between 2009 and 2011, as they have done since 2007. However, the survival rates for Phase III and beyond have recently shown signs of improvement with a 7-percentage-point increase in success rate between 2009 and 2011 as compared to between 2007 and 2009. These low Phase II success rates in combination with improving Phase III success rates, increasing proportions of Phase II efficacy failures and declining proportions of Phase III efficacy failures may be an indication that the industry, as a whole, is designing Phase II programmes that are able to support early termination decisions and thereby avoiding a number of costly Phase III failures.

John Arrowsmith and Philip Miller are at Thomson Reuters, 77 Hatton Garden, London EC1N 8JS, UK.

Correspondence to P.M.

e-mail:philip.miller@thomsonreuters.com

The authors declare no competing financial interests.

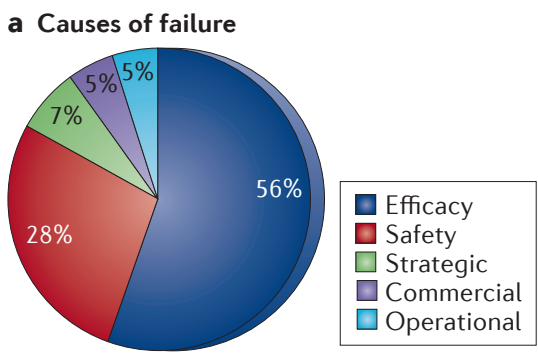

Failure by therapeutic area

b Phase II failures

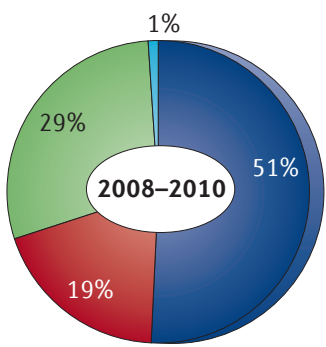

Phase III and submission failures

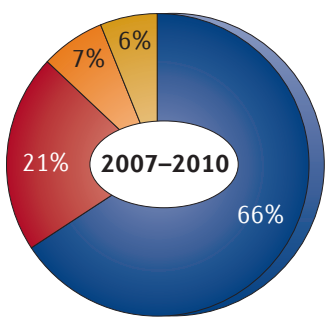

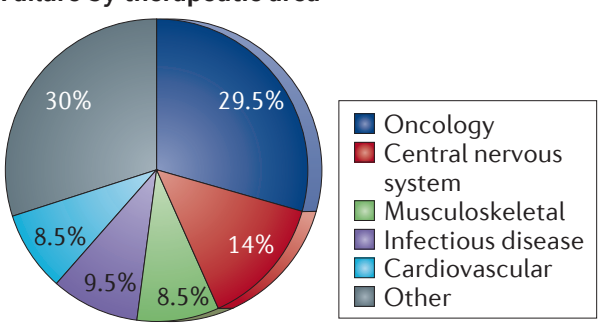
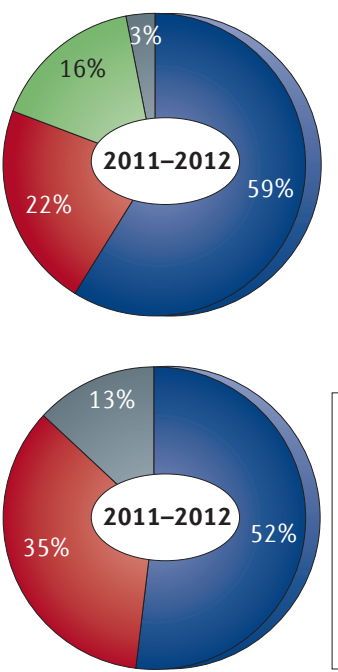

Efficacy Safety Strategic $\square$ Pharmacokinetics/ bioavailability $\square$ Other $\square$ Financial and/or Not disclosed commercial

Figure 1 | Trends in attrition rates. a | Of the 148 failures between Phase II and submission in 2011 and 2012, reasons were reported for 105; the majority of failures were due to lack of efficacy, as shown on the left. On the right, the 105 reported failures are broken down according to therapeutic area. $\mathbf{b}$ |Comparison of the reasons for failures in Phase II and Phase III trials in 2011 and 2012 with those in earlier periods that we reported previously (see main text for details). Data are from Thomson Reuters, Drugs of Today ${ }^{\circledR}$ Prous Science S.A. 